\title{
Chemometric Comparison and Classification of Some Essential Oils Extracted from Plants Belonging to Apiaceae and Lamiaceae Families Based on Their Chemical Composition and Biological Activities
}

\author{
Cristina Anamaria Semeniuc 1,*(1), Maria-Ioana Socaciu 2 (D), Sonia Ancuţa Socaci 2 (1), \\ Vlad Mureșan 1,* (D), Melinda Fogarasi ${ }^{1}$ and Ancuţa Mihaela Rotar 2 (iD \\ 1 Department of Food Engineering, University of Agricultural Sciences and Veterinary Medicine Cluj-Napoca, \\ 3-5 Mănăştur St., 400372 Cluj-Napoca, Romania; melinda.nagy@usamvcluj.ro \\ 2 Department of Food Science, University of Agricultural Sciences and Veterinary Medicine Cluj-Napoca, \\ 3-5 Mănăştur St., 400372 Cluj-Napoca, Romania; maria-ioana.socaciu@usamvcluj.ro (M.-I.S.); \\ sonia.socaci@usamvcluj.ro (S.A.S.); anca.rotar@usamvcluj.ro (A.M.R.) \\ * Correspondence: cristina.semeniuc@usamvcluj.ro (C.A.S.); vlad.muresan@usamvcluj.ro (V.M.); \\ Tel.: +40-264-596-384 (C.A.S. \& V.M.)
}

Academic Editors: Maria Daglia, Simone Carradori and Annabella Vitalone

Received: 25 July 2018; Accepted: 25 August 2018; Published: 5 September 2018

\begin{abstract}
This study is focused on the comparison and classification of parsley, lovage, basil, and thyme essential oils (EOs) based on their chemical composition, total phenolic content, antioxidant and antibacterial activities by using appropriate chemometric methods: Principal component analysis (PCA) and hierarchical cluster analysis (HCA). The results showed that parsley, lovage, and thyme EOs are rich in monoterpene hydrocarbons, but basil EO is rich in oxygenated monoterpenes and phenylpropanoids, and that both PCA and HCA separated essential oils into two main groups of which one contains two sub-groups. $\beta$-Phellandrene was the major component identified in parsley and lovage EOs, estragole was the major component in basil EO, and p-cymene was the major component in thyme EO. Thyme EO showed the highest level of total phenolics, the highest antioxidant capacity, and exhibited the stronger antibacterial activity, results that were emphasized by both chemometric methods used. Among tested essential oils, the one of parsley was distinguished by a low total TPC, weak antioxidant activity, and weak antibacterial activity against $S$. enteritidis (ATCC 13076); lovage EO by low TPC, weak antioxidant activity, but moderate antibacterial activity; and basil EO by low TPC, moderate antioxidant activity, and weak antibacterial activity against L. monocytogenes (ATCC 19114).
\end{abstract}

Keywords: essential oils; chemical composition; total phenolic content; antioxidant activity; antibacterial activity; chemometrics

\section{Introduction}

Essential oils are aromatic and volatile liquids distilled from different plant materials [1]. Numerous studies have shown that essential oils possess antioxidant, antibacterial, antifungal, and nematicidal activities, with their chemical compounds being responsible for these properties [2-13]. Plant essential oils constituents are mainly grouped in two distinct chemical classes: Terpenoids (monoterpene hydrocarbons, sesquiterpene hydrocarbons, and their oxygenated derivatives) and phenylpropanoids (phenols and phenol ethers) [14]. Both terpenoid and phenylpropanoid families comprise phenolic compounds which are considered to be mainly responsible for the antioxidant 
activity of essential oils [5,15]. In general, the climate conditions, geographical region, and several other factors directly influence the relative composition of essential oils [16].

Due to negative perceptions of synthetic preservatives among consumers, the interest in essential oils focusing on their application in food preservation has grown considerably in the last few years [1]. Essential oils added to edible products, either by direct mixing or in edible coatings and active packaging may stand as a valid alternative to prolong shelf-life and prevent autoxidation [15]. For this purpose, the food industry requires large amounts of essential oils. Vegetable raw materials (mainly leaves) used for large-scale extraction of essential oils are usually purchased from different suppliers. In their dry state they are more stable during storage and are therefore available throughout the year.

Our previous study [8] has shown that essential oils extracted from dried leaves of parsley, lovage, basil, and thyme possess antimicrobial properties. For a better understanding of their active compounds and mechanisms of action, a further in-depth study is required. Consequently, the present work aimed to determine any relationship between the chemical constituents, total phenolic content, antioxidant capacity, and antibacterial activity of parsley, lovage, basil, and thyme essential oils (EOs) extracted from dried leaves, in order to highlight the best potential of each one for future applications in the food industry. To the best of our knowledge, this is the first study that illustrates the strengths and weaknesses of these essential oils regarding their biological activities by using chemometric methods.

\section{Results}

\subsection{Yield and Chemical Composition of Essential Oils}

The highest essential oil concentration, as calculated on the basis of dry plant weight $(v / w)$, was recorded in leaves of thyme $(2.20 \%, v / w)$, followed by basil $(0.40 \%, v / w)$, lovage $(0.28 \%, v / w)$, and parsley $(0.16 \%, v / w)$. The volatile compounds detected by ITEX-GC/MS analysis in the four essential oils with their percentage composition are summarized in Table 1.

Table 1. Volatile compounds detected in essential oils.

\begin{tabular}{|c|c|c|c|c|c|c|c|}
\hline Crt. No. & Compound & $\mathrm{CC}$ & RT (min) & \multicolumn{4}{|c|}{ RC (\%) } \\
\hline 1 & Methyl 2-methylbutanoate & C5 & 4.763 & - & - & - & 2.34 \\
\hline 3 & 4-Nonene & $\mathrm{C} 5$ & 8.550 & 0.07 & - & - & - \\
\hline 4 & $\alpha$-Thujene & $\mathrm{C} 1$ & 9.666 & 0.05 & 0.26 & 0.19 & 2.04 \\
\hline 5 & $\alpha$-Pinene & $\mathrm{C} 1$ & 9.924 & 6.49 & 3.63 & 1.92 & 3.81 \\
\hline 7 & Sabinene & $\mathrm{C} 1$ & 11.502 & 0.42 & 1.39 & 1.06 & - \\
\hline 8 & $\beta$-Pinene & $\mathrm{C} 1$ & 11.668 & 2.78 & 0.89 & 2.57 & 0.58 \\
\hline 9 & 2-Octen-1-ol, (E)- & $\mathrm{C} 5$ & 11.835 & - & - & - & 0.32 \\
\hline 10 & $\beta$-Myrcene & $\mathrm{C} 1$ & 12.185 & 12.71 & 11.36 & 1.37 & 5.41 \\
\hline 11 & $\alpha$-Phellandrene & $\mathrm{C} 1$ & 12.817 & 2.93 & 1.53 & - & 0.35 \\
\hline 12 & n.i. & C6 & 13.269 & 0.09 & - & - & 3.50 \\
\hline 17 & $\beta$-Phellandrene & $\mathrm{C} 1$ & 13.908 & 32.44 & 53.89 & - & - \\
\hline 18 & trans- $\beta$-Ocimene & $\mathrm{C} 1$ & 14.064 & 0.13 & 7.00 & - & - \\
\hline 19 & cis- $\beta$-Ocimene & $\mathrm{C} 1$ & 14.486 & 0.30 & - & 0.36 & - \\
\hline 20 & $\gamma$-Terpinene & $\mathrm{C} 1$ & 14.956 & 0.68 & 0.78 & 0.41 & 18.56 \\
\hline 21 & n.i. & C6 & 15.469 & - & - & 0.09 & - \\
\hline 22 & Terpinolene & $\mathrm{C} 1$ & 16.023 & 3.76 & 1.53 & - & 0.10 \\
\hline 23 & Fenchone & $\mathrm{C} 3$ & 16.190 & - & - & 0.22 & - \\
\hline 24 & 2,6-Dimethylstyrene & $\mathrm{C} 1$ & 16.226 & 6.43 & - & - & - \\
\hline
\end{tabular}


Table 1. Cont.

\begin{tabular}{|c|c|c|c|c|c|c|c|}
\hline \multirow{2}{*}{ Crt. No. } & \multirow{2}{*}{ Compound } & \multirow{2}{*}{$\mathrm{CC}$} & \multirow{2}{*}{ RT (min) } & \multicolumn{4}{|c|}{ RC (\%) } \\
\hline & & & & Parsley EO & Lovage EO & Basil EO & Thyme EO \\
\hline 25 & Linalool acetate & C3 & 16.644 & 0.08 & 0.81 & 19.80 & 0.56 \\
\hline 26 & 1,3,8-p-Menthatriene & $\mathrm{C} 1$ & 17.127 & 13.93 & - & - & - \\
\hline 27 & Fenchol & C3 & 17.429 & - & - & 0.34 & - \\
\hline 28 & Camphor & C3 & 18.530 & - & - & 0.24 & - \\
\hline 29 & Menthone & C3 & 18.861 & 0.06 & - & - & - \\
\hline 30 & Isoborneol & $\mathrm{C} 3$ & 19.506 & - & - & - & 0.11 \\
\hline 31 & trans-4-Thujanol & C3 & 19.846 & - & - & 0.54 & - \\
\hline 32 & $\alpha$-Terpineol & $\mathrm{C} 3$ & 20.419 & - & 1.96 & 0.48 & - \\
\hline 33 & Estragole & $\mathrm{C} 4$ & 20.518 & - & - & 40.93 & - \\
\hline 34 & Anisole & $\mathrm{C} 4$ & 21.690 & - & - & - & 0.19 \\
\hline 35 & Carvone & C3 & 22.236 & 0.52 & 0.13 & - & - \\
\hline 36 & Bornyl acetate & $\mathrm{C} 3$ & 23.767 & - & - & 0.14 & - \\
\hline 37 & Anethole & $\mathrm{C} 4$ & 23.807 & 0.79 & 0.30 & - & - \\
\hline 38 & Thymol & C3 & 23.951 & - & - & 0.29 & 8.72 \\
\hline 39 & Phenol, 2-ethyl-4,5-dimethyl- & C5 & 24.240 & - & - & - & 0.35 \\
\hline 40 & $\beta$-Terpinyl acetate & C3 & 25.973 & 0.24 & 12.73 & - & - \\
\hline 41 & n.i. & C6 & 27.027 & 0.13 & - & - & - \\
\hline 42 & Eugenol methyl ether & C4 & 27.856 & - & - & 0.29 & - \\
\hline 43 & Caryophyllene & C2 & 28.583 & 0.43 & - & 0.70 & 0.16 \\
\hline \multirow[t]{2}{*}{44} & $\alpha$-Bergamotene & $\mathrm{C} 2$ & 29.003 & - & - & 0.92 & - \\
\hline & TOTAL & & & 100.0 & 100.0 & 100.0 & 100.0 \\
\hline
\end{tabular}

CC—chemical class; C1-monoterpene hydrocarbons; C2—sesquiterpene hydrocarbons; C3-oxygenated monoterpenes; $\mathrm{C} 4$-phenylpropanoids; $\mathrm{C} 5$-other compounds; C6-unidentified compounds; RT-retention time; RC-relative content of peak area; n.i.: not identified.

Parsley essential oil: Twenty-three compounds, representing $99.8 \%$ of the total detected constituents, were identified in the essential oil of parsley and grouped on the basis of their chemical structure into six classes (monoterpene hydrocarbons-C1, sesquiterpene hydrocarbons-C2, oxygenated monoterpenes-C3, phenylpropanoids-C4, other compounds-C5, and unidentified compounds-C6). The most abundant constituents were monoterpene hydrocarbons $(97.2 \%)$, followed by oxygenated monoterpenes $(0.9 \%)$, phenylpropanoids $(0.8 \%)$, others $(0.5 \%)$, sesquiterpene hydrocarbons $(0.4 \%)$, and unidentified compounds $(0.2 \%)$. The major components identified in parsley EO were $\beta$-phellandrene $(32.4 \%), 1,3,8-p$-menthatriene $(13.9 \%), 1,8$-cineole $(12.5 \%), \beta$-myrcene $(12.7 \%), \alpha$-pinene $(6.5 \%)$, and 2,6-dimethylstyrene $(6.4 \%)$, all of them belonging to the monoterpene hydrocarbons class. 1,3,8- $p$-Menthatriene is a characteristic volatile constituent of parsley leaves that contribute to the parsley aroma [17]. A similar aromatic profile was found by Petropoulos et al., 2004 [18] in essential oil extracted from dried leaves of Greek parsley at the first sowing and growth stage with $\beta$-phellandrene, 1,3,8-p-menthatriene, $\beta$-myrcene, and $\alpha, p$-dimethylstyrene being the major components. In a study on essential oil from dried leaves of Estonian parsley, Vokk et al., 2011 [19] have noticed myristicin to be the major constituent, followed by $\beta$-phellandrene, 1,3,8-p-menthatriene, and $\beta$-myrcene.

Lovage essential oil: Eighteen compounds were identified in the essential oil of lovage representing $100 \%$ of the total detected constituents. The most abundant constituents were monoterpene hydrocarbons $(83.6 \%)$, followed by oxygenated monoterpenes $(15.6 \%)$, others $(0.5 \%)$, and phenylpropanoids $(0.3 \%)$. The major components identified in lovage EO were $\beta$-phellandrene $(53.9 \%)$, $\beta$-terpinyl acetate $(12.7 \%), \beta$-myrcene $(11.4 \%)$, and trans- $\beta$-ocimene $(7.0 \%)$; the $\beta$-terpinyl acetate belongs to the oxygenated monoterpenes class and the rest of them to monoterpene hydrocarbons. The study of Bylaiteù et al., 1998 [20] has evidenced $\alpha$-terpinyl acetate as the major compound of essential oil extracted from dried leaves of Lithuanian lovage, followed by $\beta$-phellandrene, and Z-ligustilide.

Basil essential oil: Twenty-four compounds were identified in the essential oil of basil representing $99.9 \%$ of the total detected constituents. The most abundant constituents were oxygenated monoterpenes $(46.4 \%)$, followed by phenylpropanoids (41.2\%), monoterpene hydrocarbons $(10.7 \%)$, sesquiterpene hydrocarbons $(1.6 \%)$, and unidentified compounds $(0.1 \%)$. The major components identified in basil EO were estragole (40.9\%), 1,8-cineole (24.3\%), and linalool acetate (19.8\%), first 
of them belonging to phenylpropanoids and the others to oxygenated monoterpenes. In a study on essential oil extracted from dried leaves of Polish basil, Calín-Sánchez et al., 2012 [21] have found phenylpropanoids as the majority class, followed by monoterpenoids, sesquiterpenes, monoterpenes, sesquiterpenoids, aldehydes, and alcohols with methyleugenol, eugenol, 1,8-cineole, and linalool being the major components.

Thyme essential oil: Nineteen compounds were identified in the essential oil of thyme representing $96.5 \%$ of the total detected constituents. The most abundant constituents were monoterpene hydrocarbons $(83.5 \%)$, followed by oxygenated monoterpenes $(9.7 \%)$, unidentified compounds $(3.5 \%)$, others $(3.0 \%)$, sesquiterpene hydrocarbons $(0.2 \%)$, and phenylpropanoids $(0.2 \%)$. The major components identified in thyme EO were $p$-cymene $(49.8 \%), \gamma$-terpinene $(18.6 \%)$, thymol $(8.7 \%)$, and $\beta$-myrcene (5.4\%); the thymol belongs to the oxygenated monoterpenes class and the rest of them to monoterpene hydrocarbons.

A number of studies have focused on the chemical composition of essential oil extracted from dried leaves of thyme. Contrary to our study, Jamali et al., 2013 [22] have found oxygenated monoterpenes as the main constituents in Moroccan essential oil, followed by monoterpene hydrocarbons, sesquiterpene hydrocarbons, and oxygenated sesquiterpenes; the major components identified were carvacrol, $\gamma$-terpinene, $p$-cymene, and $\alpha$-pinene. Borugă et al., 2014 [4] have identified thymol, $\gamma$-terpinene, and $p$-cymene as the major components of essential oil from thyme cultivated in Romania. In another study, Calín-Sánchez et al., 2013 [23] have identified thymol, $\gamma$-terpinene, $p$-cymene, caryophyllene, and $\alpha$-terpinene as the major constituents of Polish essential oil. Agili, 2014 [3] has reported thymol, $\gamma$-terpinene, $p$-cymene, and carvacrol as the major constituents of essential oil extracted from thyme collected from Saudi Arabian markets. The main constituents found by Soto-Mendívil et al., 2006 [24] in essential oil extracted from thyme collected from Mexican markets were borneol, thymol, carvacrol methyl ether, camphene, $\alpha$-humulene, and carvacrol.

\subsection{Total Phenolic Content and Antioxidant Activity of Essential Oils}

Table 2 shows the values for total phenolic content (TPC) and Trolox equivalent antioxidant capacity (TEAC) of essential oils. The highest TPC was found in thyme EO $(22.5 \pm 1.69 \mathrm{mg}$ GAE $\left.100 \mu \mathrm{L}^{-1} \mathrm{EO}\right)$, followed by essential oil of lovage $\left(3.0 \pm 0.15 \mathrm{mg} \mathrm{GAE} 100 \mu \mathrm{L}^{-1} \mathrm{EO}\right)$, basil $\left(2.9 \pm 0.13 \mathrm{mg} \mathrm{GAE} 100 \mu \mathrm{L}^{-1} \mathrm{EO}\right)$, and parsley ( $\left.2.2 \pm 0.05 \mathrm{mg} \mathrm{GAE} 100 \mu \mathrm{L}^{-1} \mathrm{EO}\right)$. Phenolics are a class of compounds that act as free radical scavengers and are responsible for the antioxidant activity of essential oils [2,5]. The highest antioxidant capacity was noticed in essential oil extracted from thyme $\left(197.0 \pm 0.23 \mu \mathrm{M}\right.$ TE $\left.\mathrm{mL}^{-1} \mathrm{EO}\right)$, followed by the one of basil $\left(105.0 \pm 1.09 \mu \mathrm{M} \mathrm{TE} \mathrm{mL}^{-1} \mathrm{EO}\right)$, parsley $\left(39.4 \pm 0.02 \mu \mathrm{M} \mathrm{TE} \mathrm{mL}{ }^{-1} \mathrm{EO}\right)$, and lovage $\left(35.9 \pm 0.27 \mu \mathrm{M} \mathrm{TE} \mathrm{mL}^{-1} \mathrm{EO}\right)$.

It is well known that phenolic compounds act as antioxidants [25,26], but they are not fully responsible for the antioxidant activity of essential oils. In a recent study, Ferreira et al., 2017 [7] have reported an enhanced antioxidant activity of essential oils rich in oxygenated monoterpenes. In the current study, the level of total phenolics in basil EO was proximate to that found in parsley and lovage EOs; however, its antioxidant activity was approximately 3 times higher. Since oxygenated monoterpenes $(46.4 \%)$ prevailed in basil EO, we first suspected that this class of compounds is the major contributor to its antioxidant activity. However, no significant correlation between TEAC and oxygenated monoterpenes-C3 was found. In contrast, there was a positive correlation ( $\mathrm{r}=0.999$; $p<0.05)$ between TEAC and phenylpropanoids-C4. These findings show that phenylpropanoids $(41.2 \%)$ are in fact mainly responsible for the antioxidant activity of basil EO. 
Table 2. Total phenolic contents (TPC) and antioxidant activities (TEAC) of essential oils.

\begin{tabular}{ccc}
\hline Essential Oil & TPC $\left(\mathbf{m g}\right.$ GAE $\mathbf{1 0 0} \mu \mathbf{L}^{-\mathbf{1}}$ EO) & TEAC $\left(\boldsymbol{\mu M}\right.$ TE $\mathbf{~ m L}^{-\mathbf{1}}$ EO) \\
\hline Parsley EO & $2.2 \pm 0.05$ & $39.4 \pm 0.02$ \\
Lovage EO & $3.0 \pm 0.15$ & $35.9 \pm 0.27$ \\
Basil EO & $2.9 \pm 0.13$ & $105.0 \pm 1.09$ \\
Thyme EO & $22.5 \pm 1.69$ & $197.0 \pm 0.23$ \\
\hline
\end{tabular}

Values are results of three replicates.

\subsection{Antibacterial Activity of Essential Oils}

Table 3 summarizes the results of minimum inhibitory concentration and minimum bactericidal concentration tests. There was a strong positive correlation $(r=0.976 ; p<0.0001)$ between values obtained using the two tests since the minimum inhibitory concentrations (MICs) and minimum bactericidal concentrations (MBCs) were similar, except those of parsley EO against L. monocytogenes. The lower the MIC/MBC value, the higher the antibacterial activity of an essential oil. Of the four essential oils tested, basil EO has proved to be more effective against $S$. enteritidis than L. monocytogenes and the others conversely. The results show that thyme EO was the most bacteriostatic/bactericidal against $S$. enteritidis, followed equally by lovage and basil EOs, and then by parsley EO. Thyme EO was also the most bacteriostatic/bactericidal against L. monocytogenes, followed instead by essential oils of lovage, parsley, and basil. The essential oil of parsley showed similar MIC and MBC values against $S$. enteritidis (22.68 $\mu \mathrm{L} \mathrm{EO} \mathrm{mL} \mathrm{m}^{-1}$ ); a MIC value of $5.14 \mu \mathrm{L} \mathrm{EO} \mathrm{mL} L^{-1}$ and $\mathrm{MBC}$ value of $10.80 \mu \mathrm{L} \mathrm{EO} \mathrm{mL} \mathrm{m}^{-1}$ against L. monocytogenes. Essential oils of lovage, basil, and thyme revealed similar bacteriostatic and bactericidal activities against $S$. enteritidis $\left(10.80 \mu \mathrm{L} \mathrm{EO} \mathrm{mL} L^{-1}, 10.80 \mu \mathrm{L} \mathrm{EO} \mathrm{mL} L^{-1}\right.$ and $0.56 \mu \mathrm{LEO} \mathrm{mL}{ }^{-1}$, respectively) and L. monocytogenes $\left(2.45 \mu \mathrm{LEO} \mathrm{mL}^{-1}, 22.68 \mu \mathrm{L} \mathrm{EO} \mathrm{mL}^{-1}\right.$ and $0.13 \mu \mathrm{L} \mathrm{EO} \mathrm{mL}{ }^{-1}$, respectively). Jamali et al., 2013 [22] have reported levels of $270 \mu \mathrm{g} \mathrm{EO} \mathrm{mL}^{-1}$ for MIC and $540 \mu \mathrm{g} \mathrm{EO} \mathrm{mL}{ }^{-1}$ for MBC in essential oil extracted from dried leaves of thyme tested against L. monocytogenes; an antibacterial activity much lower than the one found in the current study.

Table 3. Minimum inhibitory (MIC) and minimum bactericidal (MBC) concentrations of essential oils.

\begin{tabular}{ccccc}
\hline Essential Oil & \multicolumn{2}{c}{ S. Enteritidis/ATCC 13076 } & \multicolumn{2}{c}{ L. Monocytogenes/ATCC 19114 } \\
\hline Parsley EO & $22.68 \pm 0.0$ & $22.68 \pm 0.0$ & $5.14 \pm 0.0$ & $10.80 \pm 0.0$ \\
Lovage EO & $10.80 \pm 0.0$ & $10.80 \pm 0.0$ & $2.45 \pm 0.0$ & $2.45 \pm 0.0$ \\
Basil EO & $10.80 \pm 0.0$ & $10.80 \pm 0.0$ & $22.68 \pm 0.0$ & $22.68 \pm 0.0$ \\
Thyme EO & $0.56 \pm 0.0$ & $0.56 \pm 0.0$ & $0.13 \pm 0.0$ & $0.13 \pm 0.0$ \\
\hline
\end{tabular}

Values are results of three replicates. Gentamicin's MBCs, which were identical to the MICs, were $0.11 \pm 0.0$ for S. enteritidis (ATCC 13076) and $0.05 \pm 0.0 \mu \mathrm{g} \mathrm{GE} \mathrm{mL}{ }^{-1}$ for L. monocytogenes (ATCC 19114).

\section{Discussion}

Comparison and Classification of Essential Oils Based on Their Chemical Composition, Total Phenolic Content, Antioxidant and Antibacterial Activities

Chemical PCA and HCA analysis of all compounds. The chemical constituents of parsley, lovage, basil and thyme EOs were subjected to principal component analysis (PCA) in order to better visualize and identify their grouping trends. The PCA biplot (Figure 1a) indicates high similarities among parsley and lovage EOs (upper-right plot), characterized by $\beta$-phellandrene (compound No. 17 in Table 1 and Figure 1a) and $\beta$-myrcene (No. 10). The first principal component (PC1) explained $53 \%$ of the total variance while the second one (PC2) showed a further $41 \%$. Basil and thyme EOs instead were positioned very distinctly based on their characteristics constituents: Estragole (No. 33), 1,8-cineole (No. 16), and linalool acetate (No. 25) for basil EO (bottom-left plot), respectively $p$-cymene (No. 14), $\gamma$-terpinene (No. 20), and thymol (No. 38) for thyme EO (upper-left plot). Similar to PCA, the hierarchical cluster analysis (HCA) based on the Euclidean distance between 
groups (Figure 1b) indicated three groups (A, B, and C) of essential oils; parsley and lovage EOs formed one group (group A), while basil and thyme EOs were individual distinctly separated in group $B$ and group $C$, respectively.

Chemical PCA and HCA analyses of compound class: In this case, PCA and HCA (Figure 1c,d) revealed interesting and somehow surprising results with lovage and thyme EOs forming 1st sub-group (A1) and parsley EO the 2nd one (A2) of the same cluster (group A). Basil EO formed another cluster (group B). The PCA biplot of essential oils based on their chemical compound classes (Figure 1c) showed a great chemical resemblance between lovage and thyme EOs (down-right plot), characterized by similar ratios of monoterpene hydrocarbons- $\mathrm{C} 1$ and oxygenated monoterpenes- $\mathrm{C} 3(83.6 \% \mathrm{C} 1$ and $15.6 \% \mathrm{C} 3$, respectively $83.5 \% \mathrm{C} 1$ and $9.7 \%$ C3). PC1 horizontal axis explained $99 \%$ while PC2 vertical axis explained $1 \%$ of the total variance. On the same side of the axes (upper-right plot), parsley EO revealed higher ratio $\mathrm{C} 1: \mathrm{C} 3$ than thyme and lovage EOs such as $97.2 \% \mathrm{C} 1$ and $0.9 \% \mathrm{C} 3$. On the other side of the axes (upper-left plot), basil EO showed a much lower level of monoterpene hydrocarbons-C1 $(10.7 \%)$ and higher levels of oxygenated monoterpenes-C3 (46.4\%) and phenylpropanoids-C4 $(41.2 \%)$ than parsley, lovage, and thyme EOs.

PCA and HCA analysis of total phenolic content and antioxidant activity: It was interesting to note that performing PCA and HCA based only on total phenolic contents and antioxidant capacities (Figure 2a,b), a clearer image of essential oils characteristics has been revealed. The PCA biplot of essential oils based on their TPC and TEAC (Figure 2a) also confirmed a great similarity between parsley and lovage EOs (upper-left plot); these were clustered (Figure 2b) during PCA in the 1st sub-group (A1) of the first cluster (group A) with low TPC and weak antioxidant activity. PC1 horizontal axis explained $100 \%$ of the total variance. Basil EO (down-right of biplot in Figure 2a) formed the 2nd sub-group (A2) of the same cluster as was characterized by low TPC but moderate antioxidant activity. With the highest level of TPC and stronger antioxidant activity, thyme EO (upper-right plot in Figure 2b) was grouped into a second cluster (group B).

PCA and HCA analysis of MIC and MBC. PCA and HCA (Figure 2c,d) indicate high similarities among thyme EO and gentamicin (upper-left plot in Figure 2c; sub-group A1 in Figure 2d) that showed the stronger antibacterial activity against both tested strains. Lovage EO was plotted close to them but in the bottom-left quarter of the chart as it exhibited moderate antibacterial activity; it formed the 2nd sub-group (A2) of the same cluster (group A). PC1 horizontal axis explained $71 \%$ of the total variance while the vertical axis explained a further $28 \%$. Other two distinct clusters were formed by parsley (group B) and basil EOs (group C), each one showing different antibacterial properties, parsley EO (bottom-right plot in Figure 2c) being less efficient against S. enteridis while basil EO (upper-right plot in Figure 2c) against L. monocytogenes. 


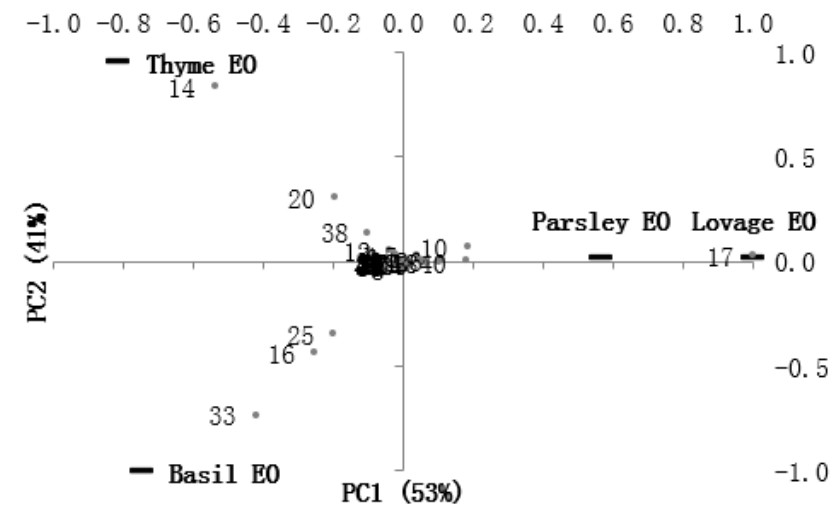

(a)

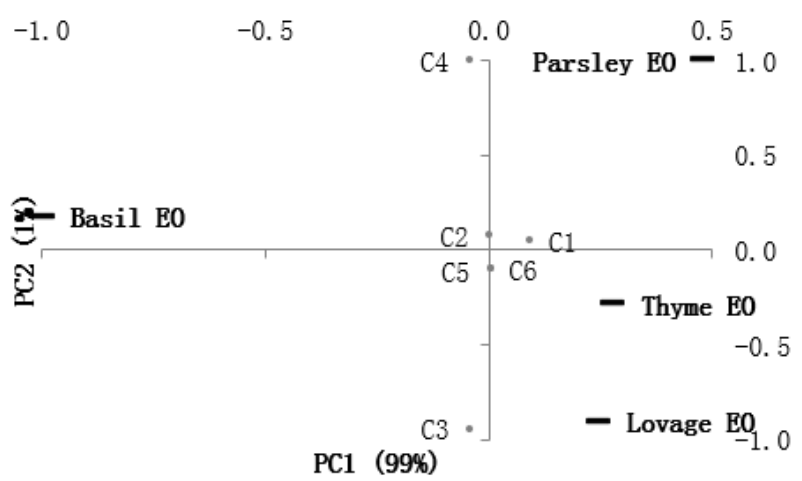

(c)

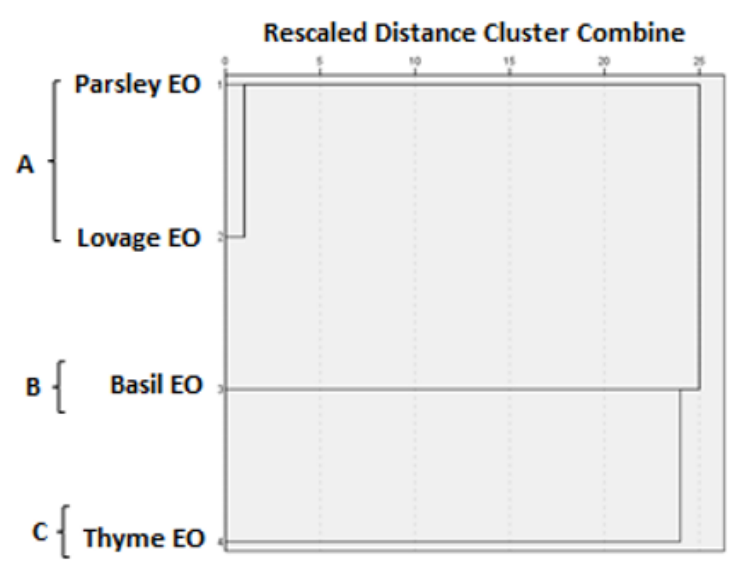

(b)

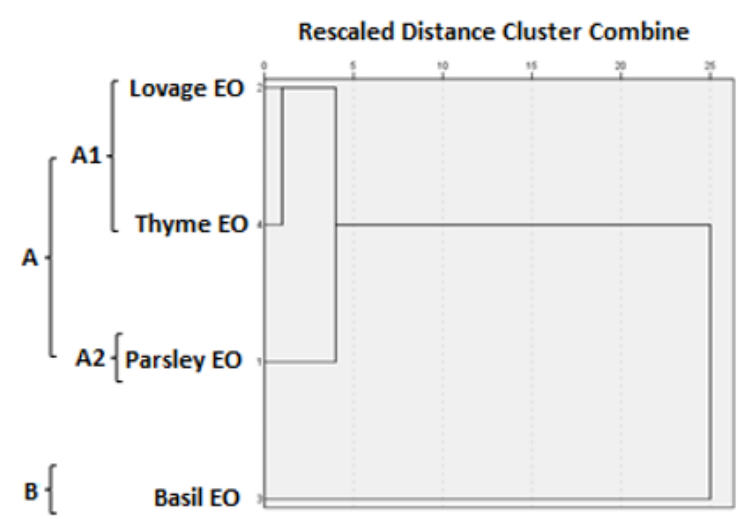

(d)

Figure 1. PCA and HCA performed on essential oil volatile compounds and their chemical classes. (a) The PCA biplot of essential oils based on their individual volatile compounds; (b) HCA dendrogram of essential oils based on their individual volatile compounds; (c) The PCA biplot of essential oils based on their chemical compound classes; (d) HCA dendrogram of essential oils based on their chemical compound classes. The numbers of chemical compounds are presented in Table 1; C1—monoterpene hydrocarbons; C2—sesquiterpene hydrocarbons; C3—oxygenated monoterpenes; C4—phenylpropanoids; C5—other compounds, and C6-unidentified compounds. 


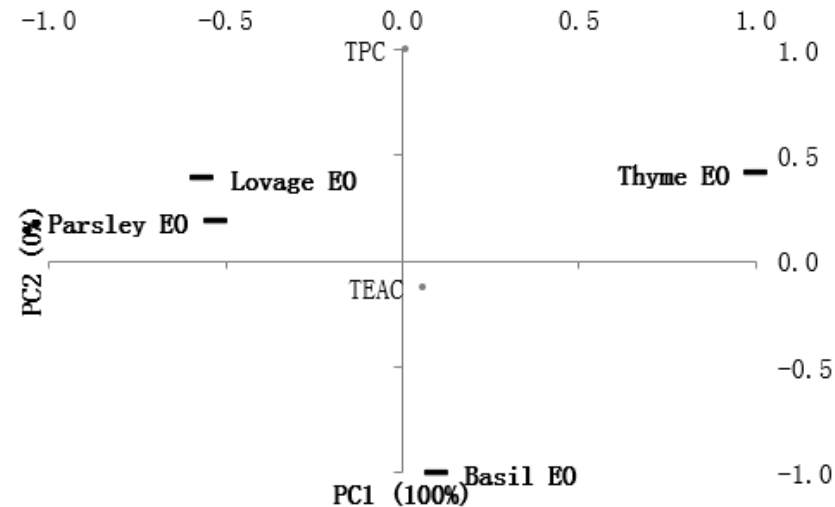

(a)

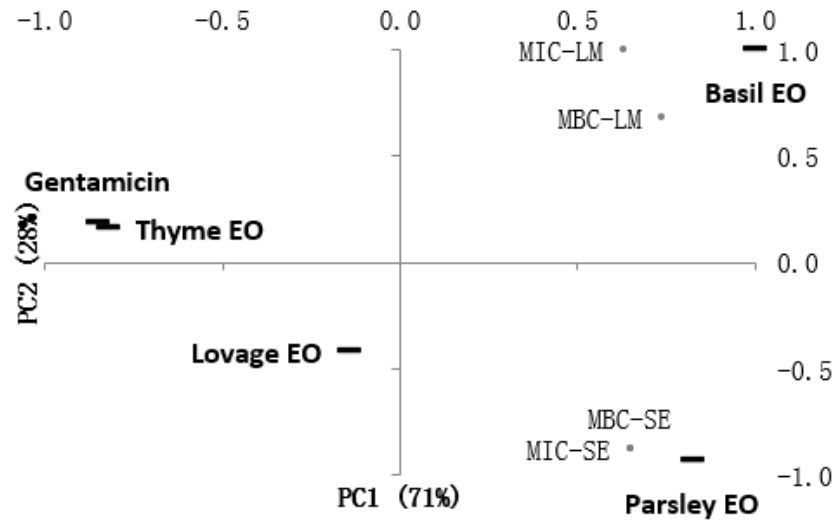

(c)

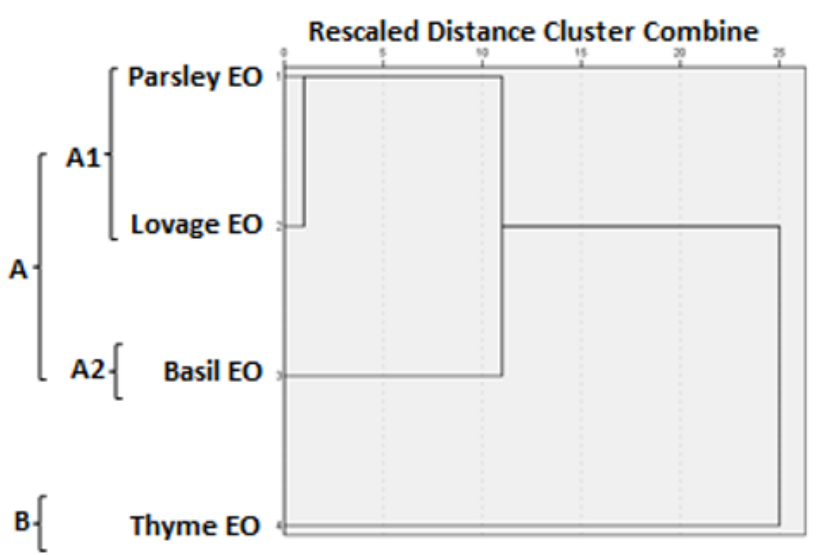

(b)

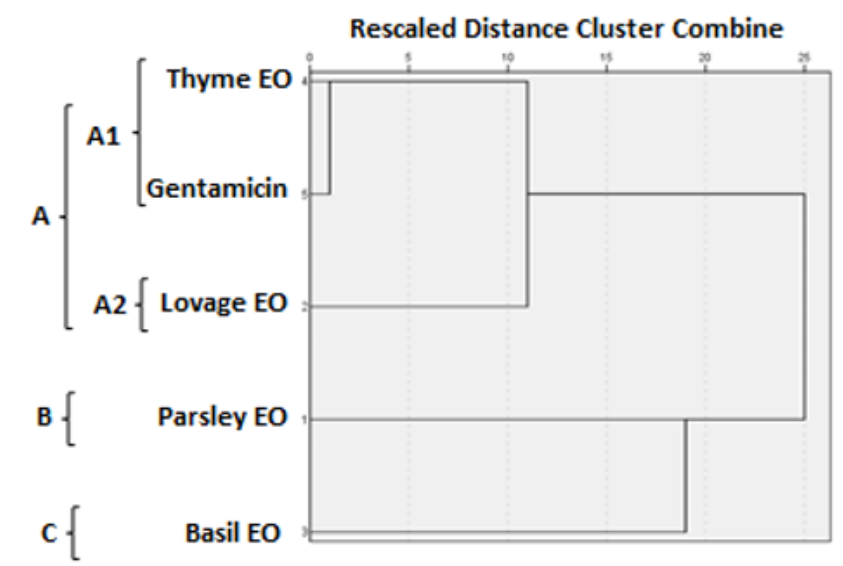

(d)

Figure 2. PCA and HCA performed on TPC and TEAC of essential oils. (a) The PCA biplot of essential oils based on their TPC and TEAC; (b) HCA dendrogram of essential oils based on their TPC and TEAC; (c) The PCA biplot of essential oils based on their MICs and MBCs; (d) HCA dendrogram of essential oils based on their MICs and MBCs. TPC—-total phenolic content; TEAC—Trolox equivalent antioxidant capacity; MIC—minimum inhibitory concentration; MBC—-minimum bactericidal concentration; LM-L. monocytogenes; SE-S. enteritidis. 


\section{Materials and Methods}

\subsection{Experimental Design}

The current study was conducted to determine the chemical composition of four essential oils extracted from herbs belonging to the families Apiaceae (parsley and lovage) and Lamiaceae (basil and thyme), their total phenolic content (TPC), antioxidant activity (TEAC), as well as minimum inhibitory and bactericidal concentrations (MIC/MBC) against two of the most common bacteria responsible for food poisoning (Salmonella enteritidis and Listeria monocytogenes).

\subsection{Plant Materials and Essential Oils Extraction}

Commercially available leaves of parsley, lovage, basil, and thyme, in the dried state, were purchased from a company that markets food ingredients (Solina Group, Alba Iulia, Romania, http: / www.solinagroup.ro). Essential oils were obtained by hydrodistillation, dried leaves (50 g) of each plant material being extracted for $3 \mathrm{~h}$ with $750 \mathrm{~mL}$ distilled water in a Clevenger-type apparatus (S.C. Energo-Metr S.R.L., Odorheiu Secuiesc, Romania). The essential oils were dried over anhydrous sodium sulphate and stored at $4{ }^{\circ} \mathrm{C}$ until analysis. The extraction yield was calculated as the volume of essential oil $(\mathrm{mL})$ per dried leaves weight $(\mathrm{g})$ and multiplied by 100 [8].

\subsection{ITEX/GC-MS Analysis of Volatile Constituents}

Qualitative ITEX/GC-MS analysis of the volatile compounds was performed using an analytical technique based on in-tube extraction (ITEX), as previously reported by our group [25]. Sample of $1 \mu \mathrm{L}$ volume was measured into a sealed-cap headspace vial $(20 \mathrm{~mL})$ and maintained on continuous agitation at $50{ }^{\circ} \mathrm{C}$ for $10 \mathrm{~min}$. The adsorption (5 strokes) of volatile compounds from the gaseous phase of the essential oil was performed by a Combi PAL AOC-5000 autosampler (CTC Analytics, Zwingen, Switzerland) with a headspace syringe ITEX-II equipped with a microtrap (ITEX-2TrapTXTA, Tenax TA 80/100 mesh, Switzerland). The analytes were released by thermal desorption into the injection port of the GC-MSQP2010 system (Shimadzu, Kyoto, Japan); the microtrap was then flash-heated with $\mathrm{N}_{2}$.

A Zebron ZB-5 ms capillary column $(30 \mathrm{~m} \times 0.25 \mathrm{~mm}$ i.d. $\times 0.25 \mu \mathrm{m}$ film thickness; Phenomenex, Torrance, CA, USA) was used for the chromatographic separation of volatile compounds. The column oven temperature program was set as follows: From $40{ }^{\circ} \mathrm{C}$ (kept at this temperature for $2 \mathrm{~min}$ ) to $160{ }^{\circ} \mathrm{C}$ at $4{ }^{\circ} \mathrm{C} / \mathrm{min}$, then raised to $240{ }^{\circ} \mathrm{C}$ at $15^{\circ} \mathrm{C} / \mathrm{min}$ (kept at this temperature for $5 \mathrm{~min}$ ). The ion source, injector, and interface temperatures were set at $250{ }^{\circ} \mathrm{C}$. Helium was used as carrier gas, the constant flow rate being $1 \mathrm{~mL} / \mathrm{min}$. The split ratios were 1:200 for parsley EO, 1:250 for lovage and basil EOs, and 1:300 for thyme EO. The MS mode used was Electron Impact (EI) ionization, while a mass range from 40 to $650 \mathrm{~m} / \mathrm{z}$ was scanned.

The volatile compounds were tentatively identified by comparing their mass spectra with those in the NIST27 and NIST147 libraries (considering a minimum similarity of 85\%) and by retention indices obtained from www.pherobase.com [27] or www.flavornet.org [28] (for columns with a similar stationary phase to ZB-5 ms). The results were expressed as a percentage of the total peaks area.

\subsection{Determination of Total Phenolic Content}

The total phenolic content was measured using the method described by Socaci et al., 2014 [29]. One hundred microliters of test sample (dilution of essential oil with methanol—parsley EO:MeOH, 1:50 (v/v); lovage EO:MeOH, 1:50 (v/v); basil EO:MeOH, 1:50 (v/v); and thyme EO:MeOH, 1:250 (v/v)) were transferred into a $16 \mathrm{~mL}$ glass bottle with a rubber stopper. Then, $6 \mathrm{~mL}$ of distilled water and $0.5 \mathrm{~mL}$ of $2 \mathrm{~N}$ Folin-Ciocalteu phenol reagent were added and immediately vortexed (Vortex V-1 Plus, Biosan Ltd., Riga, Latvia). After $4 \mathrm{~min}, 1.5 \mathrm{~mL}$ of $0.71 \mathrm{M}$ sodium carbonate aqueous solution and $1.9 \mathrm{~mL}$ distilled water were added to the mixture. The incubation of the test sample was carried out in the dark at room temperature for $2 \mathrm{~h}$. The absorbance value was read at $725 \mathrm{~nm}$ against blank sample using a 
double beam UV-VIS spectrophotometer (PharmaSpec UV-1700, Shimadzu, Kyoto, Japan). The blank sample was prepared with methanol and treated identically to the test sample. Three readings per sample were taken. The result was expressed in mg gallic acid equivalents (GAE) $100 \mu \mathrm{L}^{-1} \mathrm{EO}$.

\subsection{Determination of Antioxidant Activity}

The Trolox equivalent antioxidant capacity assay was used to measure the antioxidant activity of essential oils; it was performed according to the method described by Thaipong et al., 2006 [30]. The stock solution was prepared by mixing equal volumes of $7.4 \mathrm{mM}$ ABTS aqueous solution and $2.6 \mathrm{mM}$ potassium persulfate aqueous solution and allowing them to react for $12 \mathrm{~h}$ at room temperature in the dark. The working solution was prepared by mixing $1 \mathrm{~mL}$ of stock solution with $60 \mathrm{~mL}$ of methanol to obtain an absorbance of $1.1 \pm 0.02$ units at $734 \mathrm{~nm}$. One hundred and fifty microliters of test sample (dilution of essential oil with methanol—parsley EO:MeOH, 1:100 (v/v); lovage EO:MeOH, 1:100 (v/v); basil EO:MeOH, 1:300 (v/v); and thyme EO:MeOH, 1:500 $(v / v)$ ) were transferred into a $16 \mathrm{~mL}$ glass bottle with a rubber stopper, then $2850 \mu \mathrm{L}$ of ABTS working solution were added and vortexed. The incubation of the test sample was carried out in the dark, at room temperature for $2 \mathrm{~h}$. The absorbance value was read at $734 \mathrm{~nm}$ against methanol using a double beam UV-VIS spectrophotometer (PharmaSpec UV-1700, Shimadzu, Kyoto, Japan). The blank sample was prepared with methanol and treated identically to the test sample. Three readings per sample were taken. The absorbance value of the test sample is extracted from the absorbance value of the blank sample; the final result was expressed in $\mu \mathrm{M}$ Trolox equivalent (TE) $\mathrm{mL}^{-1} \mathrm{EO}$.

\subsection{Bacterial Strains}

The following microorganisms were tested: Salmonella enteritidis (ATCC 13076, Microbiologics Inc., St. Cloud, MN, USA) and Listeria monocytogenes (ATCC 19114, Microbiologics Inc., St. Cloud, MN, USA). Each strain was grown into a test tube containing $10 \mathrm{~mL}$ sterile nutrient broth (Oxoid Ltd., Basingstoke, Hampshire, England) at $37^{\circ} \mathrm{C}$ for $24 \mathrm{~h}$ in the case of S. enteritidis and at $37^{\circ} \mathrm{C}$ for $48 \mathrm{~h}$ in the case of L. monocytogenes. The purity of the inoculum was confirmed by plating on appropriate selective media and microscopic examination of the Gram-stained smear (Optika microscope, B-252, M.A.D. Apparecchiature Scientifiche, Milan, Italy). A loopful of inoculum was transferred by streaking onto a selective medium: XLD agar (Oxoid Ltd., Basingstoke, Hampshire, England) for S. enteritidis and Palcam agar base (Oxoid Ltd., Basingstoke, Hampshire, England) with added Palcam selective supplement for L. monocytogenes. Plates were incubated at $37^{\circ} \mathrm{C}$ for $24 \mathrm{~h}$ in the case of $S$. enteritidis and at $37^{\circ} \mathrm{C}$ for $48 \mathrm{~h}$ in the case of L. monocytogenes. Bacterial morphology was confirmed by optical microscopy. Several colonies were collected with a sterile inoculating loop, transferred into sterile saline solution $\left(8.5 \mathrm{~g} \mathrm{~L}^{-1}\right)$, and adjusted to match the turbidity of a McFarland 0.5 standard $\left(1.5 \times 10^{8} \mathrm{CFU} \mathrm{mL}^{-1}\right)$ [31]. Then, three serial 10 -fold dilutions $\left(10^{7}, 10^{6}\right.$, and $\left.10^{5} \mathrm{CFU} \mathrm{mL}{ }^{-1}\right)$ were prepared using the sterile saline solution as diluent.

\subsection{Minimum Inhibitory Concentration Test (MIC)}

The test was performed according to the method described by Semeniuc et al., 2017 [8]. Eight parts $50 \%$ ethanol solution and one part Tween 80 were mixed with one part of the essential oil [32]. Sterile nutrient broth $(100 \mu \mathrm{L})$ and diluted essential oil $(100 \mu \mathrm{L})$ were added into the first well of a 96-well microtiter plate. Serial 11-fold dilutions were obtained by pipetting $100 \mu \mathrm{L}$ from well to well (on row), while $100 \mu \mathrm{L}$ were discarded from the last well of the row. Next, a volume of $10 \mu \mathrm{L}$ inoculum $\left(1.5 \times 10^{5} \mathrm{CFU} \mathrm{mL}{ }^{-1}\right)$ was added to each well. Concentrations ranging from 0.01 to $47.62 \mu \mathrm{L} \mathrm{EO} \mathrm{mL}^{-1}$ were thus reached. For the negative control, eight parts 50\% ethanol and one part Tween 80 were mixed with one part of the saline solution, while gentamicin $\left(0.4 \mathrm{mg} \mathrm{mL}^{-1}\right.$ in saline solution) was considered the positive control. Microplates were incubated at $37^{\circ} \mathrm{C}$ for $21 \mathrm{~h}$ in the case of S. enteritidis and at $37^{\circ} \mathrm{C}$ for $45 \mathrm{~h}$ in the case of L. monocytogenes. A volume of $20 \mu \mathrm{L}$ resazurin aqueous solution $\left(0.2 \mathrm{mg} \mathrm{mL}^{-1}\right)$ was added to each well. Microplates were subsequently incubated at $37^{\circ} \mathrm{C}$ for $2 \mathrm{~h}$ in the 
case of S. enteritidis and at $37^{\circ} \mathrm{C}$ for $23 \mathrm{~h}$ in the case of L. monocytogenes. The lowest concentration that retained the blue color was considered the concentration that completely inhibited bacterial growth (MIC). Three replicates were run for each essential oil. The result was expressed in $\mu \mathrm{L} \mathrm{EO} \mathrm{mL}^{-1}$, respectively $\mu$ g gentamicin $(\mathrm{GE}) \mathrm{mL}^{-1}$.

\subsection{Minimum Bactericidal Concentration Test (MBC)}

To determine the minimum bactericidal concentration, $10 \mu \mathrm{L}$ of the dilution representing the MIC and the more concentrated two dilutions were plated on the appropriate selective media (XLD agar for S. enteritidis and Palcam agar base with added Palcam selective supplement for L. monocytogenes) using a Drigalski spatula and incubated for $48 \mathrm{~h}$ at $37^{\circ} \mathrm{C}$. A colony counter (Colony Star 8500, Funke Gerber, Berlin, Germany) was used to determine the relative number of bacterial colonies. The lowest concentration of the antimicrobial agent causing negative growth (fewer than three colonies) was considered to be the concentration that completely killed bacteria [32]. The result was expressed in $\mu \mathrm{L} \mathrm{EO} \mathrm{mL}^{-1}$.

\subsection{Statistical Analysis}

Hierarchical clustering (HCA) and correlation analysis were carried out using IBM SPSS Statistics software, version 19.0 (IBM Corp., Armonk, New York, NY, USA). Cluster analysis was run using the complete linkage method. Correlation analysis between was performed via Pearson's correlation. Principal component analysis (PCA) was carried out using Unscrambler software, version 9.7 (CAMO Software AS, Oslo, Norway).

\section{Conclusions}

Monoterpenes hydrocarbons were the most abundant chemical compounds in parsley, lovage, and thyme EOs, while in basil, oxygenated monoterpenes and phenylpropanoids prevailed. Regarding the antibacterial activity, all tested essential oils showed inhibitory and bactericidal effects on both bacteria; most of them were more effective against L. monocytogenes (ATCC 19114) but basil EO was more effective against $S$. enteritidis (ATCC 13076). The highest amount of total phenolics and antioxidant capacity were found in thyme EO; this essential oil also exhibited the stronger antibacterial activity. Parsley EO instead had a low total phenolic content (TPC), weak antioxidant activity, and weak antibacterial activity against $S$. enteritidis (ATCC 13076); lovage EO by low TPC, weak antioxidant activity, and moderate antibacterial activity; basil EO by low TPC, moderate antioxidant activity, and weak antibacterial activity against L. monocytogenes (ATCC 19114).

Author Contributions: Conceptualization, C.A.S., V.M. and A.M.R.; Formal analysis, C.A.S. and V.M.; Funding acquisition, C.A.S. and V.M.; Investigation, C.A.S., M.-I.S., S.A.S. and M.F.; Methodology, C.A.S.; Supervision, C.A.S. and V.M.; Writing—original draft, C.A.S.; Writing—review \& editing, V.M.

Funding: This research was funded by a grant of the Romanian Ministry of Research and Innovation, CCCDI-UEFISCDI, project number PN-III-P2-2.1-CI-2017-0034, within PNCDI III and by a grant of Ministry of Research and Innovation, CNCS - UEFISCDI, project number PN-III-P1-1.1-PD-2016-0113, within PNCDI III.

Acknowledgments: We are grateful for the administrative and financial support received from the University of Agricultural Sciences and Veterinary Medicine Cluj-Napoca, Romania.

Conflicts of Interest: The authors declare no conflict of interest.

\section{References}

1. Hyldgaard, M.; Mygind, T.; Meyer, R.L. Essential oils in food preservation: Mode of action, synergies, and interactions with food matrix components. Front. Microbiol. 2012, 3, 12. [CrossRef] [PubMed]

2. Alizadeh, A.; Alizadeh, O.; Amari, G.; Zare, M. Essential oil composition, total phenolic content, antioxidant activity and antifungal properties of Iranian Thymus daenensis subsp. daenensis Celak. as in influenced by ontogenetical variation. J. Essent. Oil Bearing Plants 2013, 16, 59-70. [CrossRef] 
3. Agili, F.A. Chemical composition, antioxidant and antitumor activity of Thymus vulgaris L. essential oil. Middle-East J. Sci. Res. 2014, 21, 1670-1676. [CrossRef]

4. Borugă, O.; Jianu, C.; Mişcă, C.; Goleț, I.; Gruia, A.T.; Horhat, F.G. Thymus vulgaris essential oil: Chemical composition and antimicrobial activity. J. Med. Life 2014, 7, 56-60. [PubMed]

5. Marín, I.; Sayas-Barberá, E.; Viuda-Martos, M.; Navarro, C.; Sendra, E. Chemical composition, antioxidant and antimicrobial activity of essential oils from organic fennel, parsley, and lavender from Spain. Foods 2016, 5, 18. [CrossRef] [PubMed]

6. Dhifi, W.; Bellili, S.; Jazi, S.; Bahloul, N.; Mnif, W. Essential oils' chemical characterization and investigation of some biological activities: A critical review. Medicines 2016, 3, 25. [CrossRef] [PubMed]

7. Ferreira, D.D.; Nora, F.M.D.; Lucas, B.N.; Menezes, C.R.D.; Cichoski, A.J.; Giacomelli, S.R.; Wagner, R.; Barin, J.S. Oxygen introduction during extraction and the improvement of antioxidant activity of essential oils of basil, lemon and lemongrass. Ciênc Rural. 2017, 47, e20170045. [CrossRef]

8. Semeniuc, C.A.; Pop, C.R.; Rotar, A.M. Antibacterial activity and interactions of plant essential oil combinations against Gram-positive and Gram-negative bacteria. J. Food Drug Anal. 2017, 25, 403-408. [CrossRef] [PubMed]

9. Gómez-Rincón, C.; Langa, E.; Murillo, P.; Valero, M.S.; Berzosa, C.; López, V. Activity of tea tree (Melaleuca alternifolia) essential oil against L3 larvae of Anisakis simplex. Biomed. Res. Int. 2014, 2014, 549510. [CrossRef] [PubMed]

10. Valero, A.; Romero, M.C.; Gómez-Mateos, M.; Hierro, I.; Navarro, M.C. Natural products: Perspectives in the pharmacological treatment of gastrointestinal anisakiasis. Asian Pac. J. Trop. Med. 2015, 8, 612-617. [CrossRef] [PubMed]

11. Giarratana, F.; Muscolino, D.; Ziino, G.; Giuffrida, A.; Marotta, S.M.; Lo Presti, V.; Chiofalo, V.; Panebianco, A. Activity of Tagetes minuta Linnaeus (Asteraceae) essential oil against L3 Anisakis larvae type 1. Asian Pac. J. Trop. Dis. 2017, 10, 461-465. [CrossRef] [PubMed]

12. Gómez-Mateos Pérez, M.; Navarro Moll, C.; Merino Espinosa, G.; Valero López, A. Evaluation of different Mediterranean essential oils as prophylactic agents in anisakidosis. Pharm. Biol. 2017, 55, 456-461. [CrossRef] [PubMed]

13. Giarratana, F.; Muscolino, D.; Ziino, G.; Lo Presti, V.; Rao, R.; Chiofalo, V.; Giuffrida, A.; Panebianco, A. Activity of Catmint (Nepeta cataria) essential oil against Anisakis larvae. Trop. Biomed. 2017, 34, 22-31.

14. Zuzarte, M.; Salgueiro, L. Essential oils chemistry. In Bioactive Essential Oils and Cancer; de Sousa, D.P., Ed.; Springer International Publishing AG: Cham, Switzerland, 2015; pp. 19-62, ISBN 78-3-319-19144-7.

15. Amorati, R.; Foti, M.C.; Valgimigli, L. Antioxidant activity of essential oils. J. Agric. Food. Chem. 2013, 61, 10835-10847. [CrossRef] [PubMed]

16. Si, L.; Chen, Y.; Han, X.; Zhan, Z.; Tian, S.; Cui, Q.; Wang, Y. Chemical composition of essential oils of Litsea cubeba harvested from its distribution areas in China. Molecules 2012, 17, 7057-7066. [CrossRef] [PubMed]

17. Charles, D.J. Parsley. In Handbook of Herbs and Spices, 1st ed.; Peter, K.V., Ed.; Woodhead Publishing Limited: Cambridge, UK, 2004; Volume 2, pp. 230-242, ISBN 978-1-85573-721-1.

18. Petropoulos, S.A.; Daferera, D.; Akoumianakis, C.A.; Passam, H.C.; Polissiou, M.G. The effect of sowing date and growth stage on the essential oil composition of three types of parsley (Petroselinum crispum). J. Sci. Food Agric. 2004, 84, 1606-1610. [CrossRef]

19. Vokk, R.; Lõugas, T.; Mets, K.; Kravets, M. Dill (Anethum graveolens L.) and parsley (Petroselinum crispum (Mill.) Fuss) from Estonia: Seasonal differences in essential oil composition. Agron. Res. 2011, 9, 515-520.

20. Bylaiteù, E.; Venskutonis, R.P.; Roozen, J.P. Influence of harvesting time on the composition of volatile components in different anatomical parts of lovage (Levisticum officinale Koch.). J. Agric. Food. Chem. 1998, 46, 3735-3740. [CrossRef]

21. Calín-Sánchez, Á.; Lech, K.; Szumny, A.; Figiel, A.; Carbonell-Barrachina, Á.A. Volatile composition of sweet basil essential oil (Ocimum basilicum L.) as affected by drying method. Food Res. Int. 2012, 48, 217-225. [CrossRef]

22. Jamali, C.A.; Kasrati, A.; Bekkouche, K.; Hassani, L.; Wohlmuth, H.; Leach, D.; Abbad, A. Phenological changes to the chemical composition and biological activity of the essential oil from Moroccan endemic thyme (Thymus maroccanus Ball). Ind. Crops Prod. 2013, 49, 366-372. [CrossRef]

23. Calín-Sánchez, Á.; Figiel, A.; Lech, K.; Szumny, A.; Carbonell-Barrachina, Á.A. Effects of drying methods on the composition of thyme (Thymus vulgaris L.) essential oil. Drying Technol. 2013, 31, 224-235. [CrossRef] 
24. Soto-Mendívil, E.A.; Moreno-Rodríguez, J.F.; Estarrón-Espinosa, M.; García-Fajardo, J.A.; Obledo-Vázquez, E.A. Chemical composition and fungicidal activity of the essential oil of Thymus vulgaris against Alternaria citri. e-Gnosis 2006, 4, 1-7.

25. Semeniuc, C.A.; Rotar, A.; Stan, L.; Pop, C.R.; Socaci, S.; Mireşan, V.; Muste, S. Characterization of pine bud syrup and its effect on physicochemical and sensory properties of kefir. CyTA-J. Food. 2016, 14, $213-218$. [CrossRef]

26. Mehla, N.; Sindhi, V.; Josula, D.; Bisht, P.; Wani, S.H. An introduction to antioxidants and their roles in plant stress tolerance. In Reactive Oxygen Species and Antioxidant Systems in Plants: Role and Regulation under Abiotic Stress; Khan, M.I.R., Khan, N.A., Eds.; Springer: Singapore, 2017; pp. 1-23, ISBN 978-981-10-5254-5.

27. The Pherobase: Database of Pheromones and Semiochemicals. Available online: www.pherobase.com (accessed on 20 June 2018).

28. Flavornet and Human Odor Space. Available online: www.flavornet.org (accessed on 20 June 2018).

29. Socaci, S.A.; Socaciu, C.; Mureşan, C.; Fărcaş, A.; Tofană, M.; Vicaş, S.; Pintea, A. Chemometric discrimination of different tomato cultivars based on their volatile fingerprint in relation to lycopene and total phenolics content. Phytochem. Anal. 2014, 25, 161-169. [CrossRef] [PubMed]

30. Thaipong, K.; Boonprakob, U.; Crosby, K.; Cisneros-Zevallos, L.; Byrne, D.H. Comparison of ABTS, DPPH, FRAP, and ORAC assays for estimating antioxidant activity from guava fruit extracts. J. Food Compos. Anal. 2006, 19, 669-675. [CrossRef]

31. McFarland, J. The nephelometer: An instrument for estimating the number of bacteria in suspensions used for calculating the opsonic index and for vaccines. JAMA 1907, 49, 1176-1178. [CrossRef]

32. Preuss, H.G.; Echard, B.; Enig, M.; Brook, I.; Elliott, T.B. Minimum inhibitory concentrations of herbal essential oils and monolaurin for gram-positive and gram-negative bacteria. Mol. Cell. Biochem. 2005, 272, 29-34. [CrossRef] [PubMed]

Sample Availability: Samples of the compounds are not available from the authors.

(C) 2018 by the authors. Licensee MDPI, Basel, Switzerland. This article is an open access article distributed under the terms and conditions of the Creative Commons Attribution (CC BY) license (http://creativecommons.org/licenses/by/4.0/). 